\title{
Electromagnetically Induced Left Handedness in Optically Excited Four-Level Atomic Media
}

\author{
Quentin Thommen and Paul Mandel \\ Optique Nonlinéaire Théorique, Université Libre de Bruxelles, Campus Plaine CP 231, 1050 Bruxelles, Belgium
}

(Received 18 July 2005; published 6 February 2006)

\begin{abstract}
We show that left-handed properties can be electromagnetically induced in a general four-level atomic medium for a finite spectral range. We use an electric (magnetic) atomic transition as an electric (magnetic) resonator to modify the permittivity (permeability), both at the same frequency. The implementation of the four-level model is carried out in atomic hydrogen and neon. In each case the existence of left-handed properties is predicted inside an experimentally reachable domain of parameters.
\end{abstract}

PACS numbers: 42.50.Gy, 42.25.Bs, 78.20.Ci

The propagation of electromagnetic waves in matter is characterized by the frequency-dependent relative dielectric permittivity $\varepsilon_{r}$ and magnetic permeability $\mu_{r}$. Their product defines the index of refraction: $\varepsilon_{r} \mu_{r}=n^{2}$. Lefthanded media are characterized by negative real parts of $\varepsilon_{r}, \mu_{r}[1]$. It has been shown that in this case the negative root must be used for the index of refraction $n=-\sqrt{\varepsilon_{r} \mu_{r}}$ [1]. Left-handed media have recently attracted significant attention since it has been discovered that a slab of lefthanded materials is able, for instance, to focus light into a spot much smaller than the wavelength, realizing a "perfect" lens [2]. Experimentally, left-handed properties in the microwave domain were obtained with a composite medium made of a periodic array alternation of split ring resonators and continuous wires [3]. Left handedness has been recently analyzed in a three-level medium [4]. However, the proposed scheme requires the conflicting demand that the middle state is involved in both a magnetic transition and an electric transition at the same frequency. An independent study of the same scheme has reached a similar conclusion [5].

In this Letter we demonstrate that a four-level medium may be left handed in a restricted domain of parameters. In our model an electric transition and a magnetic transition play the role of an electric resonator and a magnetic resonator, respectively. They involve two different pairs of states, leading to a realistic energy configuration. Explicit calculation predict the occurrence of a left-handed domain in hydrogen and neon gases with $\operatorname{Re}\left\{\varepsilon_{r}\right\}<0$ and $\operatorname{Re}\left\{\mu_{r}\right\}<0$.

Numerous works have been carried out over the past 15 years to control the index of refraction by quantum interference. They led, in particular, to electromagnetically induced transparency (EIT) [6], refractive index enhancement [7], and slow light [8]. As the magnetic transition strengths are typically 2 orders of magnitude smaller than the electric transition strengths, the permeability does not change significantly from unity as the frequency of the probe magnetic field reaches a magnetic resonance. Therefore, the modification of the index of refraction is in general due mainly to a modification of the electrical susceptibility. However, a significant change of the permeability can be obtained from a magnetic moment induced by coupled electric transitions [4]. In this Letter we combine the modification of the permeability by an induced magnetic moment with the modification of the permittivity due to an electric transition. In this way, the permeability and the permittivity can be simultaneously modified and lefthanded properties are obtained. Moreover, the absorption is reduced by EIT to increase the practicability of the scheme. After considering the general four-level scheme, we present its implementation on atomic hydrogen and neon.

We consider four atomic states having the following properties: $|1\rangle$ and $|2\rangle$ have the same parity and $\vec{\mu}_{12}=$ $\langle 1|\hat{\vec{\mu}}| 2\rangle \neq 0$, where $\hat{\vec{\mu}}$ is the magnetic dipole operator; $|3\rangle$ and $|4\rangle$ have opposite parity with $\vec{d}_{34}=\langle 3|\hat{\vec{d}}| 4\rangle \neq 0$, where $\hat{\vec{d}}$ is the electric dipole operator. As indicated in Fig. 1, we introduce three electromagnetic fields to couple the four states and describe them by their effective Rabi $\Omega_{i j}$ and optical $\omega_{a, b, c}$ frequencies. Contrary to the scheme used in [4], the electric transition $|3\rangle \rightarrow|4\rangle$ and the magnetic transition $|1\rangle \rightarrow|2\rangle$ involve different states.

For the scheme to be consistent, it is obvious from parity selection rules that not all transitions can be electric dipolar. Therefore, one of the transitions must involve either

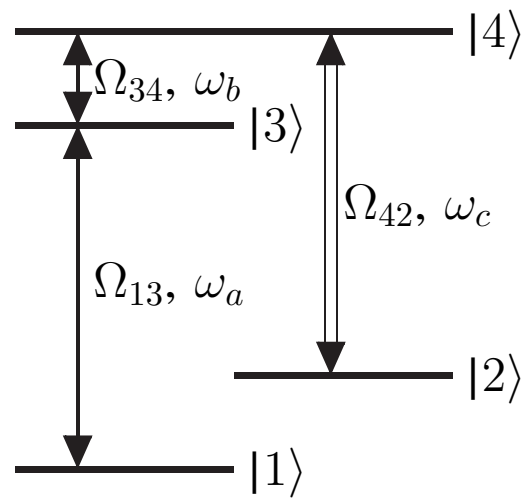

FIG. 1. Four-level medium interacting with three electromagnetic fields. 
two photons or a quadrupole. In the following, we shall always assume that the transition $|3\rangle \rightarrow|4\rangle$ is an electric dipolar transition. The Rabi frequency is then defined as $\Omega_{34}=\vec{d}_{34} \cdot \vec{E}_{b} / 2 \hbar$ where $\vec{E}_{b}$ is the amplitude of the electric field with frequency $\omega_{b}$.

The Hamiltonian of the system is $H=H_{0}+H_{i}$ where $H_{0}=\sum_{j} \epsilon_{j}|j\rangle\langle j|$ is the field-free Hamiltonian and $H_{i}$ is the interaction of the atom with the electromagnetic fields

$$
\left\langle k\left|H_{i}\right| l\right\rangle=-\frac{1}{2} \hbar \Omega_{k l} e^{-i\left(\omega_{k}-\omega_{l}\right) t},
$$

where $\omega_{1}=\omega_{a}+\omega_{b}, \omega_{2}=\omega_{c}, \omega_{3}=\omega_{b}$, and $\omega_{4}=0$. The nonvanishing Rabi frequencies $\Omega_{13}, \Omega_{34}$, and $\Omega_{42}$ are indicated in Fig. 1. The rotating-wave approximation has been used, and only resonant terms have been kept. The density matrix operator is defined by

$$
\hat{\rho}=\sum_{k, l=1}^{4} \rho_{k l} e^{i\left(\omega_{k}-\omega_{l}\right) t}|k\rangle\langle l| .
$$

The time-varying off-diagonal density matrix elements verify the evolution equations

$$
i \dot{\rho}_{k l}=\left(\delta_{k l}-i \gamma_{k l}\right) \rho_{k l}+\sum_{m=1}^{4}\left[\Omega_{m l} \rho_{k m}-\Omega_{k m} \rho_{m l}\right],
$$

where the damping rates $\gamma_{k l}$ of the coherences are related to the population decay rates $\gamma_{k k}$ through $2 \gamma_{k l}=\gamma_{k k}+$ $\gamma_{l l}$. The detunings are defined by $\hbar \delta_{k l}=\epsilon_{k}-\epsilon_{l}+$ $\hbar\left(\omega_{k}-\omega_{l}\right)$.

In this Letter we only consider the case of a strong electromagnetic coupling for the transition $|2\rangle \rightarrow|4\rangle$ and of weak electromagnetic coupling for the transitions $|1\rangle \rightarrow$ $|3\rangle$ and $|3\rangle \rightarrow|4\rangle$. The field $\vec{E}_{b}$ is the probe field. Therefore only the light shift produced by $\Omega_{42}$ is taken into account. In this way, the scheme is equivalent to the usual threelevel scheme used to electromagnetically induce transparency with a multiphotonic probe transition. In the following, the standard EIT approximations are used [9]. This includes the assumption that the populations are nearly constant. Moreover, it is assumed that the ground state $|1\rangle$ is the only state initially populated. In this framework it is easy to solve Eq. (3) in the steady state

$$
\begin{aligned}
& \rho_{12}=\frac{-\Omega_{13} \Omega_{34} \Omega_{42} \rho_{11}}{\left(\delta_{13}-i \gamma_{13}\right)\left[\left(\delta_{12}-i \gamma_{12}\right)\left(\delta_{14}-i \gamma_{14}\right)-\left|\Omega_{42}\right|^{2}\right]}, \\
& \rho_{34}=\frac{\Delta \Omega_{34}\left|\Omega_{13}\right|^{2} \rho_{11}}{\left(\delta_{13}-i \gamma_{13}\right)\left[\left(\delta_{12}-i \gamma_{12}\right)\left(\delta_{14}-i \gamma_{14}\right)-\left|\Omega_{42}\right|^{2}\right]},
\end{aligned}
$$

where

$$
\Delta=\frac{\left(\delta_{12}-i \gamma_{12}\right)\left(\delta_{32}-i \gamma_{32}\right)+\left|\Omega_{42}\right|^{2}}{\left(\delta_{34}-i \gamma_{34}\right)\left(\delta_{32}-i \gamma_{32}\right)-\left|\Omega_{42}\right|^{2}} .
$$

The key idea is to obtain an electric dipole and a magnetic dipole oscillating at the same frequency in order to modify simultaneously the dielectric permittivity and the magnetic permeability. If $\vec{\mu}_{12} \neq 0$, the coherence $\rho_{12}$ drives a magnetic dipole oscillating at frequency $\omega_{b}+$ $\omega_{a}-\omega_{c}$, while $\rho_{34}$ drives an electric dipole oscillating at frequency $\omega_{b}$. Dipole synchronization requires therefore either $\omega_{a}=\omega_{c}$ or $\omega_{c}=\omega_{a}+2 \omega_{b}$. We choose $\omega_{a}=\omega_{c}$ and will explain later on that the second option is impracticable.

The two coherences $\rho_{12}$ and $\rho_{34}$ are necessary to evaluate the dielectric and magnetic responses of the medium to the probe field $\vec{E}_{b}$ at frequency $\omega_{b}$. The classical electric dipole $\vec{P}$ is related to the mean value of the atomic dipole moment operator through $\vec{P}=\operatorname{Tr}\{\hat{\rho} \hat{\vec{d}}\}$, where Tr denote the trace. The amplitude of the electric dipole oscillating at frequency $\omega_{b}$ is $\vec{P}\left(\omega_{b}\right)=\vec{d}_{34} \rho_{43}$. The electric polarizability is a rank 2 tensor defined by its Fourier transform $\vec{P}(\omega)=\varepsilon_{0} \alpha_{e}(\omega) \vec{E}(\omega)$. In the following, we only consider the polarizability at the frequency $\omega_{b}$ of the incoming field $\vec{E}_{b}$. Therefore we drop the explicit $\omega_{b}$ dependence $\alpha_{e}\left(\omega_{b}\right) \equiv \alpha_{e}$. Moreover, we choose $\vec{E}_{b}$ parallel to the atomic dipole $\vec{d}_{34}$ so that $\alpha_{e}$ is a scalar. The electric polarizability for the field $E_{b}$ is

$$
\alpha_{e}=\frac{\left|d_{34}\right|^{2} \Delta\left|\Omega_{13}\right|^{2} \rho_{11}}{\varepsilon_{0} \hbar\left(\delta_{13}-i \gamma_{13}\right)\left[\left(\delta_{12}-i \gamma_{12}\right)\left(\delta_{14}-i \gamma_{14}\right)-\left|\Omega_{42}\right|^{2}\right]} .
$$

In the same way, the classical magnetization $\vec{M}$ is related to the mean value of the atomic dipole moment operator through $\vec{M}=\operatorname{Tr}\{\hat{\rho} \hat{\vec{\mu}}\}$. We focus, here again, on the component oscillating at frequency $\omega_{b}$ which is $\vec{M}\left(\omega_{b}\right)=$ $\vec{\mu}_{21} \rho_{12}$. The magnetizability is also a rank 2 tensor defined in the Fourier space by $\mu_{0} \vec{M}(\omega)=\alpha_{m}(\omega) \vec{B}(\omega)$. The magnetic field is related to the electric field by Maxwell's relation $\vec{B}_{b}=\vec{k}_{b} \times \vec{E}_{b} / \omega_{b}$, where $\vec{k}_{b}$ is the wavevector of the $\vec{E}_{b}$ field. If we choose $\vec{\mu}_{12}$ parallel to $\vec{k}_{b} \times \vec{E}_{b}$, the magnetizability is also a scalar and its expression is

$$
\alpha_{m}=\frac{-\mu_{0} c \mu_{21} d_{34} \Omega_{13} \Omega_{42} \rho_{11}}{\eta \hbar\left(\delta_{13}-i \gamma_{13}\right)\left[\left(\delta_{12}-i \gamma_{12}\right)\left(\delta_{14}-i \gamma_{14}\right)-\left|\Omega_{42}\right|^{2}\right]},
$$

where $\eta$ is a unitary complex number depending on the polarization of the field $\vec{E}_{b}$.

Using Eqs. (6) and (7), the relative permittivity and the relative permeability are easily obtained

$$
\varepsilon_{r}=1+N \alpha_{e}, \quad \mu_{r}=\frac{1}{1-N \alpha_{m}},
$$

where $N$ is the atomic density [10].

The largest variation of $\varepsilon_{r}\left(\mu_{r}\right)$ is expected at $\delta_{34}=0$ $\left(\delta_{12}=0\right)$. A strategy to tune simultaneously $\varepsilon_{r}$ and $\mu_{r}$ is to find a medium verifying the degeneracy condition

$$
\epsilon_{2}-\epsilon_{1}=\epsilon_{4}-\epsilon_{3}
$$


so that $\delta_{34}=\delta_{12}$. Furthermore, to reduce the absorption, we consider a range of parameters such that EIT affects the $|3\rangle \rightarrow|4\rangle$ transition. Actually, if $\delta_{32}=0$ is realized, the parameter $\Delta$ vanishes around $\delta_{12}=0$ as $\left|\Omega_{42}\right|^{2}$ is close to $\gamma_{12} \gamma_{32}$. Contrary to the standard EIT scheme where the absorption uniformly decreases as the power of the driving field increase, here it develops a minimum.

Let us consider a medium verifying the condition (9) and having all the damping rates practically equal $\gamma_{i j}=\gamma$. Let $\delta_{42}=0$ and $\left|\Omega_{42}\right|^{2}=\gamma^{2}$. Then, to first order in $\delta \equiv$ $\delta_{12} / \gamma$

$$
\begin{aligned}
\varepsilon_{r} & =1+\delta N\left|d_{34}\right|^{2}\left|\Omega_{13}\right|^{2} \rho_{11} / 2 \epsilon_{0} \hbar \gamma^{3}, \\
\mu_{r} & =\frac{1}{1-i N \mu_{0} c \mu_{21} d_{34} \Omega_{13} \rho_{11} / \eta \hbar \gamma^{2}} .
\end{aligned}
$$

Using typical values $\gamma=10^{8} \mathrm{~Hz}, \Omega_{13}=10^{7} \mathrm{~Hz},\left|d_{34}\right| \simeq$ $10^{-29} \mathrm{Cm}$, and $\mu_{12} \simeq \mu_{B}$, negative permittivity and negative permeability at $\delta=-0.1$ are obtained for a density $N>10^{25}$ at $/ \mathrm{m}^{3}$, which is perfectly realistic. Note that it is because we are interested in the vicinity of $\delta_{12}=0$ that we need to choose $\omega_{a}=\omega_{c}$.

Hence left-handed properties of a four-level medium can be electromagnetically induced provided the medium verifies the degeneracy condition (9). This condition implies a medium having an electric transition and a magnetic transition at the same frequency and is the main constraint for the implementation of the scheme in any materials. In the rest of this Letter, we show that these conditions can be verified in real atomic media.

First, we will consider the case of atomic hydrogen. In this case, the states $|1\rangle$ and $|2\rangle$ are the hyperfine sublevels of the ground state $1 S_{1 / 2}$ while $|3\rangle(|4\rangle)$ in an hyperfine sublevel of the exited state $2 P_{1 / 2}\left(2 S_{1 / 2}\right)$. The same optical field is used to drive the $|1\rangle \rightarrow|3\rangle$ and the $|2\rangle \rightarrow|4\rangle$ transitions. Since the transition $|2\rangle \rightarrow|4\rangle$ must involve two photons, an additional static electric field is used. This automatically ensures that the condition $\omega_{a}=\omega_{c}$ is verified.

The dependence of $\varepsilon_{r}$ and $\mu_{r}$ on the detuning $\delta_{12}$ is shown in Fig. 2. For the parameters we choose, left-handed properties of dense hydrogen gas are obtained for a $21 \mathrm{~cm}$ radio field inside the shaded area with bandwidth of about $0.2 \mathrm{MHz}$. The calculation is made with the following assumptions. The probe radio field is $\sigma_{-}$polarized. The static electric field is $\pi$ polarized with an intensity of $20 \mathrm{kV} / \mathrm{m}$. The static magnetic field with an intensity of $13 \mathrm{mT}$ is used to verify the degeneracy condition (9). The optical field oscillating at $123 \mathrm{~nm}$ with a power of $70 \mathrm{~mW} / \mathrm{m}^{2}$ propagates in the direction of the quantization axis (defined by the magnetic field) with a rectilinear polarization. The density is $N=2.5 \times 10^{25}$ at $/ \mathrm{m}^{3}$.

Up to this point, hydrogen seems to be a good candidate for the experimental realization of electromagnetically induced left handedness. But, in order to observe it in the

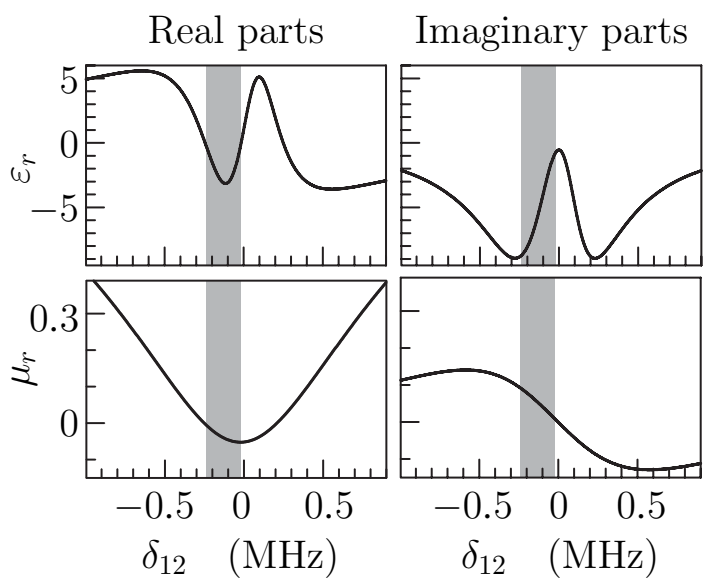

FIG. 2. Frequency dependence of the dielectric permittivity $\varepsilon_{r}$ and magnetic permeability $\mu_{r}$ of a dense hydrogen gas. Lefthanded properties are obtained in the shaded area of $0.2 \mathrm{Mhz}$ for the $21 \mathrm{~cm}$ line.

gas, we must be able to induce it on a length scale much longer than the wavelength. For hydrogen, this entails the use of a beam diameter significantly larger than $21 \mathrm{~cm}$. This is a serious obstacle for the experimental realization of left handedness in hydrogen.

In order to circumvent this difficulty, we seek an atomic species with a larger hyperfine or spin-orbit energy split for a (meta) stable state. This can be found in rare gases. Rare gases energy levels can be understood in the $(\mathrm{jL})$ coupling scheme, except for helium [11]. In particular the first exited state configuration $n p^{5}(n+1) s$ of rare gases shows a spinorbit splitting of the order of $100 \mathrm{THz}$.

Let us look at the case of neon. As a starting point, we expect all the atoms to be initially in the long-lived state of the $2 p^{5} 3 s$ configuration which is labeled $2 p^{5}\left({ }^{2} P_{3 / 2}^{o}\right) 3 s$

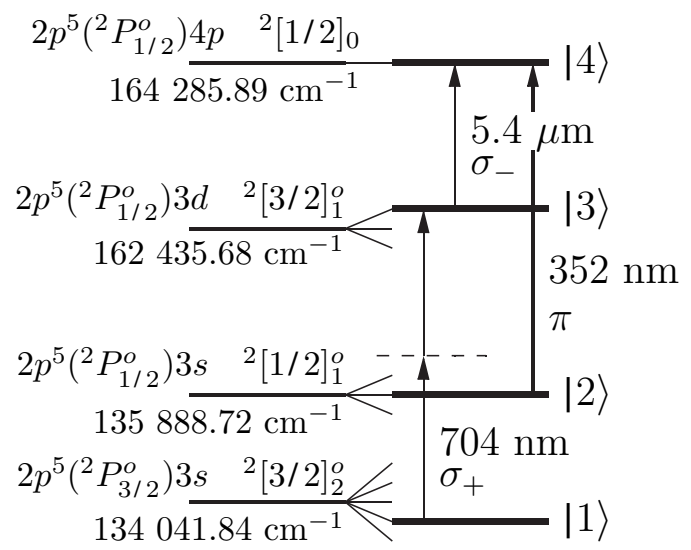

FIG. 3. Four-level scheme in neon with Zeeman effect. The neon ground sate $2 p^{6}$ is taken to be the zero point energy. Wavelength as well as polarization of the electromagnetic field used for the computation presented in Fig. 4 are indicated. The atomic parameters can be obtained from NIST [14]. 


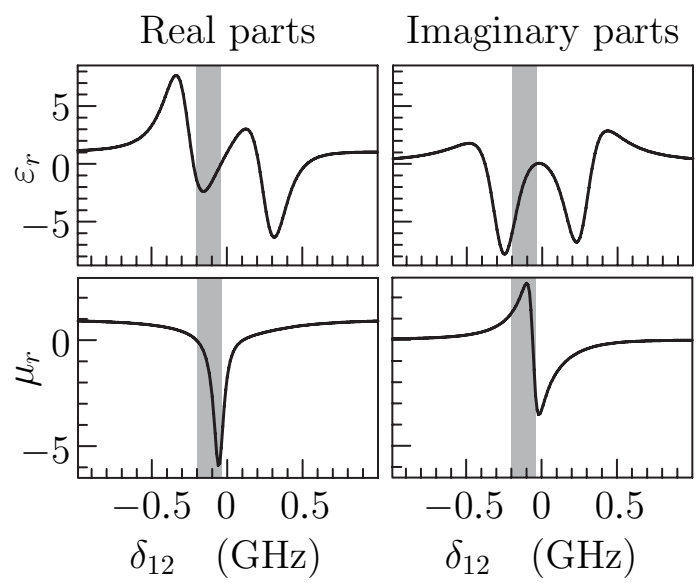

FIG. 4. Frequency dependence of the dielectric permittivity $\varepsilon_{r}$ and magnetic permeability $\mu_{r}$ of the dense neon gas. Lefthanded properties are obtained in the shaded area of about $160 \mathrm{Mhz}$.

${ }^{2}[3 / 2]_{2}^{o}$ in the $(j L)$ coupling scheme [12]. In the notation ${ }^{2}[3 / 2]_{2}^{o}$ the number $K$ in the square brackets results from coupling the $j$ angular momentum of the parent levels indicated in parentheses $\left({ }^{2} P_{3 / 2}^{o}\right)$ with the orbital angular momentum of the external electron. The subscript indicates the total angular momentum $J$, resulting from the coupling of the $K$ angular momentum and the spin of the external electron. Odd-parity levels are distinguished by the superscript $o$. Figure 3 displays the energy levels used, as well as the wavelength of the transitions. Levels $|1\rangle$ and $|2\rangle$ are fine split energy levels of the $2 p^{5} 3 s$ configuration which ensure that $\vec{\mu}_{12} \neq 0$.

As indicated in Fig. 3, levels $|1\rangle,|2\rangle$, and $|3\rangle$ have odd parity and $|4\rangle$ has even parity. The transition $|1\rangle \rightarrow|3\rangle$ involves two photons. The transition $|1\rangle \rightarrow|4\rangle$ involves an electric dipole. Figure 4 presents the computed dependence of the permittivity and the permeability on the detuning $\delta_{12}$. Neon gas shows left-handed properties in the shaded area of bandwidth about $160 \mathrm{MHz}$ for an electromagnetic field with a wavelength of $5.4 \mu \mathrm{m}$. In the calculation, we assume that the transition $|1\rangle \rightarrow|3\rangle$ is driven by a $\sigma_{+}$-polarized electric field with a wavelength of $704 \mathrm{~nm}$ and with a power of $5.1 \mathrm{~W} / \mathrm{mm}^{2}$. The transition $|2\rangle \rightarrow|4\rangle$ is driven by a $\pi$ polarized electromagnetic field with a wavelength of $352 \mathrm{~nm}$ and with a power of $77 \mathrm{~mW} / \mathrm{mm}^{2}$. The state $2 p^{5}\left({ }^{2} P_{3 / 2}^{o}\right) 3 p{ }^{2}[1 / 2]_{1}$ is used as a virtual state in the two-photon transition. The probe field is then $\sigma_{-}$polarized. The density is $N=2.5 \times$ $10^{25} \mathrm{at} / \mathrm{m}^{3}$. A small detuning $\delta_{42}=-3 \mathrm{MHz}$ is set to displace the zero of the imaginary part of $\mu_{r}$ into the area of negative real part of $\varepsilon_{r}$. In this way, the absorption is strongly reduced. The condition (9) is verified by a magnetic field of 7.14 T. For instance, for $\delta_{12}=$ $-76 \mathrm{MHz}$ we obtain $\varepsilon_{r}=-0.94-i 0.39, \mu_{r}=-4.79+$ $i 1.97$, and an index of refraction of $n=-2.30-i 0.004$. This correspond to a typical absorption length of $8 \mathrm{~mm}$. As this length is of the same order as the laser beam radius, it should be possible to observe left-handed electromagnetic properties in an atomic neon gas.

Using thermodynamic arguments, it was shown that in a left-handed passive medium the real and imaginary parts of the index of refraction must have opposite signs [13]. This sign condition is not valid here since we deal with active media.

In conclusion, we have demonstrated that left-handed electromagnetic properties can be electromagnetically induced in a four-level medium. The practicability of the scheme has been checked in a gas of hydrogen and of neon. Atomic gases are a medium of choice for a first experimental implementation of the scheme because their energy levels configurations are well known. Nevertheless this scheme needs to be applied in a solid state to realize optical devices. The degeneracy condition (9) constitutes a criterion to select promising media. Calculations along this line are in progress.

The authors acknowledge support of the Fonds National de la Recherche Scientifique (Belgium) and the Interuniversity Attraction Pole Program-Belgian Science Policy.

[1] V. G. Vesalego, Usp. Fiz. Nauk 92, 517 (1964); Sov. Phys. Usp. 10, 509 (1968).

[2] J. B. Pendry, Phys. Rev. Lett. 85, 3966 (2000).

[3] R. A. Shelby, D. R. Smith et, and S. Schultz, Science 292, 77 (2001); D. R. Smith, W. J. Padilla, D. C. Vier, S. C. Nemat-Nasser, and S. Schultz, Phys. Rev. Lett. 84, 4184 (2000).

[4] M. Ö. Oktel and Ö. E. Müstecaplıŏlu, Phys. Rev. A 70, 053806 (2004).

[5] J. Shen, Z. Ruan, and S. He, J. Zhejiang Univ. SCI 5, 1322 (2004).

[6] S. E. Harris, J. E. Field, and A. Imamoğlu, Phys. Rev. Lett. 64, 1107 (1990).

[7] M. O. Scully, Phys. Rev. Lett. 67, 1855 (1991).

[8] L. V. Hau, S.E. Harris, Z. Dutton, and C. H. Behroozi, Nature (London) 397, 594 (1999).

[9] P. Mandel, Hyperfine Interact. 135, 223 (2001).

[10] J.D. Jackson, Classical Electrodynamics (Wiley, New York, 1975). 2nd ed.

[11] R. D. Cowan, The Theory of Atomic Structure and Spectra (University of California, Berkeley, 1981).

[12] D. R. Beck, Phys. Rev. A 66, 034502 (2002).

[13] A.L. Pokrovsky and A.L. Efros, Solid State Commun. 124, 283 (2002).

[14] http://physics.nist.gov/PhysRefData. 